a non-magnetic material. Two particular features make this possible. First, the magnetic centre must be sufficiently close to the conduction channel. In this respect, the case of bis-phthalocyaninato-terbium(III) is rather peculiar, because the $\mathrm{Tb}^{3+}$ ion $\left(\mathrm{Tb}^{3+}\right.$ carries a total angular momentum, $J=6$ ) is sandwiched between two phthalocyanine ligands, and it is at least $1 \mathrm{~nm}$ away from the nanotube - too far to transfer any magnetic information. However, there is a second source of spin in this molecule, namely a $S=1 / 2$ radical delocalized over the two phthalocyanine ligands. These are likely to participate in the bond and help to spin-polarize the electron current. Second, the conduction channel must be sufficiently sensitive to the local magnetic moment. All the atoms in a single-walled carbon nanotube reside on the surface, so that a surface modification results in an alteration of the entire electronic structure. It is an extreme surface sensitivity that makes this spin valve work.

The experiments by Urdampilleta and co-workers are the first demonstration of a fully functional spin device that is entirely made of organic materials at the singlemolecule level. Their architecture adds to a number of other devices aiming at exploring spin transport in organics ${ }^{8}$. Indeed, the system is still not ideal for applications. The low operating temperature, the fact that the entire structure is rather fragile and that it cannot be manufactured with high yield, and the difficulties of performing any magnetic characterization beyond a transport measurement, are all obstacles to overcome in the future. Yet, the demonstrated possibility of manipulating spins with single molecules opens a completely new world to spintronics, where memory, logic and possibly quantum logic may be integrated. $\square$

Stefano Sanvito is at the School of Physics and the Centre for Research on Adaptive Nanostructures and Nanodevices (CRANN), Trinity College,

Dublin 2, Ireland.

e-mail:sanvitos@tcd.ie

\section{References}

1. Urdampilleta, M. et al. Nature Mater. 10, 502-506 (2011).

2. Mott, N. F. Proc. R. Soc. Lond. A 153, 699-717 (1936).

3. Baibich, M. N. Phys. Rev. Lett. 61, 2472-2475 (1988).

4. Binasch, G. Phys. Rev. B 39, 4828-4830 (1989).

5. Sanvito, S. Nature Phys. 6, 562-564 (2010).

6. Brede, J. et al. Phys. Rev. Lett. 105, 047204 (2010).

7. Barraud, C. et al. Nature Phys. 6, 615-620 (2010).

8. Sanvito, S. Chem. Soc. Rev. 40, 3336-3355 (2011).

\title{
SINK OR SWIM
}

Some of the best technology is the oldest. Sandbags have been used by armies to stop bullets since the Napoleonic Wars, because the granular medium is very efficient at absorbing the energy of the projectile and dissipating it as friction between grains.

Experiments on the shockabsorbing capabilities of granular materials generally show that a projectile penetrates only to a shallow depth before coming to rest. Military researchers hope to exploit this property in armour that might be considered a sort of 'advanced sandbag', with grains tailored for maximal stopping power - for example, arranged as tapered chains of gradually increasing or decreasing grain size, sandwiched between confining plates ${ }^{1}$.

But anyone who studies granular media knows well to take nothing for granted. For instance, they can switch between solid-like and fluid-like behaviour in response to vibration or shearing - a dangerous capability in the case of seismic hazard. Some lizards exploit that duality to actually swim through sand, using an undulatory motion to control the solid-fluid transition ${ }^{2}$.

Even bearing such subtleties in mind, a new observation of projectile motion in a granular medium by Pacheco-Vázquez and colleagues ${ }^{3}$ is remarkable. They find that an object penetrating into grains under gravity can, in some circumstances, acquire a finite terminal velocity in other words, it never comes to rest, but penetrates to (in theory) an infinite depth.

The researchers demonstrated this experimentally by dropping heavy balls (ping-pong balls filled with small steel spheres) into a deep silo of expanded polystyrene beads a few millimetres in diameter. The beads are packed loosely by first blowing air through them: the packing fraction of 0.637 sounds rather close to the random close-packed fraction of 0.64 , but in fact the bed is rather less than close-packed because of the polydispersity of bead sizes.

Balls of lighter mass come to rest within the column of grains, but above a certain mass threshold they sink all the way to the bottom. In the latter case the balls appear to reach a constant speed as they descend. This, Pacheco-Vázquez and colleagues argue, is a genuine terminal velocity.

As counter-intuitive as it might seem, the behaviour is not hard to rationalize. One of the striking properties of granular media is that, below a certain depth, the pressure reaches a constant value - in contrast to the case for ordinary liquids, where pressure continues to increase with depth. This pressure determines the friction experienced by a descending particle, and so it is possible for the drag to balance the downward force

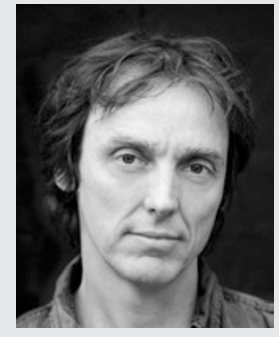

PHILIP BALL

due to gravity, just as with an object falling at terminal velocity in a normal viscous fluid.

However, these situations are not identical. Resistance to the projectile's motion is caused by the temporary formation of chains of grains in contact. This means that the force of resistance fluctuates, so that descent proceeds in a jerky, stick-slip fashion - as the researchers' simulations confirm. They end with what sounds like a warning for military sandbag engineers: in certain circumstances, they say, "we believe that self-propelled intruders in static sand may also reach terminal speeds."

References

1. Doney, R. \& Sen, S. Ordered and Highly Scalable Granular Media for Shock Mitigation ARL-TR-3612 (Army Research Laboratory, 2005).

2. Shimada, T., Kadau, D., Shinbrot, T. \& Herrmann, H. J. Phys. Rev. E 80, 020301(R) (2009).

3. Pacheco-Vázquez, F. et al. Phys. Rev. Lett. 106, 218001 (2011). 